\title{
Tricuspid valve infective endocarditis associated with aseptic meningitis: a rare presentation in a child
}

\author{
Gülsüm Alkan, M.D., Fellow ${ }^{1}$, Melike Emiroğlu M.D., Assistant Professor Doctor ${ }^{1}$, \\ Ahmet Sert M.D., Associate Professor Doctor ${ }^{2}$, Ayşe Kartal M.D., Associate Professor Doctor ${ }^{3}$ and \\ Mehmet Öc, M.D. Professor Doctor ${ }^{4}$
}

\begin{abstract}
Infective endocarditis (IE) is a rare but a potentially lifethreatening infectious disease. Atypical presentations cause delays in the diagnosis. Neurological involvement such as meningitis or meningismus, are especially common in mitral valve endocarditis, but unusual in tricuspid valve endocarditis. Although few cases of right-sided IE have been reported with neurological symptoms in adults, children have not been described in literature. A nine-year-old girl with congenital ventricular septal defect (VSD) was admitted with fever, headache and neck stiffness. Her clinical symptoms and cerebrospinal fluid findings supported the aseptic meningitis. On ceftriaxone therapy day 3 , her complaints were resolved; nine days later she was admitted with fever and neck stiffness again. Further investigation for fever source with echocardiogram revealed a tricuspid valve endocarditis. Antibiotic therapy was completed after 6 weeks. Cardiac surgery was performed for VSD and tricuspid valvular insufficiency.

Key words: aseptic meningitis, infective endocarditis, tricuspid regurgitation, ventricular septal defect, children.
\end{abstract}

http: / / dx.doi.org/10.5546/ aap.2020.eng.e22

To cite: Alkan G, Emiroğlu M, Sert A, Kartal A, Öc M. Tricuspid valve infective endocarditis associated with aseptic meningitis: a rare presentation in a child. Arch Argent Pediatr 2020;118(1):e22-e25.

a. Department of Pediatric Infectious Diseases.

b. Department of Pediatric Cardiology.

c. Department of Pediatric Neurology.

d. Department of Cardiovascular Surgery.

Selcuk University Faculty of Medicine, Konya, Turkey.

E-mail address:

Author Gülsüm Alkan: galkan-85@hotmail.com

Funding: None.

Conflict of interest: None.

Received: 12-9-2018

Accepted: 7-19-2019

\section{INTRODUCTION}

Infective endocarditis (IE) is a life-threatening infection disease of the endocardium or heart valves. Although uncommon in children, it is important the early diagnosis because of high mortality and morbidity rates. ${ }^{1}$ Approximately $35-60 \%$ of children diagnosed with IE, have congenital heart disease (CHD). Ventricular septal defect (VSD), patent ductus arteriosus, bicuspid aortic valve and tetralogy of Fallot, are high risk for IE. Children with CHD may develop endocarditis at any age, commonly on the left side. Right-sided infective endocarditis is relatively rare and frequently involves the tricuspid valve. Predominantly occurs in intravenous drug users and persons with intravascular catheters and other intravascular devices ${ }^{1,2}$

The diagnosis of IE is based on detailed history, physical examination, repeated blood cultures, and echocardiography. Osler's nodes, splinter hemorrhages, Roth spots, and Janeway lesions are rare but specific clinical signs for disease. Risk factor for endocarditis and a new murmur in any febrile child, lead to an early diagnosis. Streptococci, Staphylococci and Enterococci are the most causative organism in children. Heart failure due to vegetation on the aortic or mitral valve is the most common manifestation $^{3,4}$

Complications of IE include cardiac, neurologic, renal, musculoskeletal, and pulmonary as well as related to embolization, metastatic infection, and mycotic aneurysm. ${ }^{5}$

Neurologic sequelae are the most frequent extra cardiac complications of IE and are dominated by stroke. Although neurological complications develop in 25-70\% of adult patients, in children have been less described. Early diagnosis, antimicrobial therapy, and surgery are the cornerstones of management. ${ }^{6}$

\section{CASE REPORT}

A 9-years-old girl was admitted with fever and headache for two days. She was under follow-up for a small ( $3 \mathrm{~mm}$ in diameter) 
congenital VSD. There was no history of dental and surgical procedures. A physical examination demonstrated, body temperature of $38.5^{\circ} \mathrm{C}$, respiratory rate of $20 /$ minute, pulse rate of 110 / minute, blood pressure $100 / 60 \mathrm{mmHg}$. A loud holosystolic murmur at the lower left sternal border, positive stiff neck and Kerning sign were noticed. She was otherwise normal. She had no splinter hemorrhages, splenomegaly, or obvious focal neurologic deficits.

The laboratory data included: white blood count (WBC) $17 \times 10^{3} / \mathrm{mm}^{3}$, neutrophils $93 \%$, lymphocytes $5 \%$, hemoglobin $12.7 \mathrm{~g} / \mathrm{dL}$, platelets $225,000 / \mathrm{mm}^{3}$, erythrocyte sedimentation rate (ESR) $40 \mathrm{~mm} / \mathrm{hr}$ and C-reactive protein (CRP) $10 \mathrm{mg} / \mathrm{dL}$. The cerebrospinal fluid (CSF) cell count was 22 neutrophils $/ \mathrm{mm}^{3}$, 66 lymphocyte/ $\mathrm{mm}^{3}$ and the protein and glucose contents were $26 \mathrm{mg} / \mathrm{dL}$ and $61 \mathrm{mg} / \mathrm{dL}$, respectively. Magnetic resonance imaging was unremarkable. Ceftriaxone was started for suspected meningitis after lumber puncture. Her complaints were completely resolved on hospitalization day 3. After CSF gram stain, culture and bacterial-viral meningitis panel PCR, blood and urine cultures were all found negative, ceftriaxone was stopped, and she was discharged on hospitalization day 7 .
Two days later she was admitted again with fever $\left(39.5^{\circ} \mathrm{C}\right)$ and neck stiffness. Laboratory analysis was as follows: WBC $25 \times 10^{3} / \mathrm{mm}^{3}(92 \%$ neutrophils with toxic granulations), hemoglobin $11 \mathrm{~g} / \mathrm{dL}$, platelets 216,000/ $\mathrm{mm}^{3}$, ESR $55 \mathrm{~mm} / \mathrm{hr}$ and CRP $16 \mathrm{mg} / \mathrm{dL}$. At physical examination, there is no obvious source of fever. Her family denied to CSF examination. Further investigation for fever resource with echocardiogram showed the tricuspid valve endocarditis with a vegetation $(10,7 \times 6,6 \mathrm{~mm}$; Figure 1$)$ and VSD (3 $\mathrm{mm}$ in diameter; video clip 1) with left to right shunting was detected. Cranial computed tomography was normal. On following, her neck stiffness was resolved within 24 hours. Two consecutive blood cultures were positive for methicillin sensitive Staphylococcus aureus. Teicoplanin and gentamicin were started. Vegetation on tricuspid valve caused valvular insufficiency, so was removed by extracorporeal cardiac surgery including tricuspid valve pasty after four weeks. Antibiotics were stopped after 6 weeks, when blood cultures were found negative for two weeks. She was discharged with mild residual tricuspid regurgitation. The patient was symptom-free during one year on follow-up.

FIGURE 1. Transthorasic echocardiography showing the tricuspid valve vegetation. Vegetation, approximately $10.7 \times 6.6 \mathrm{~mm}$ in diameter, attached to the septal leaflet of tricuspid valve

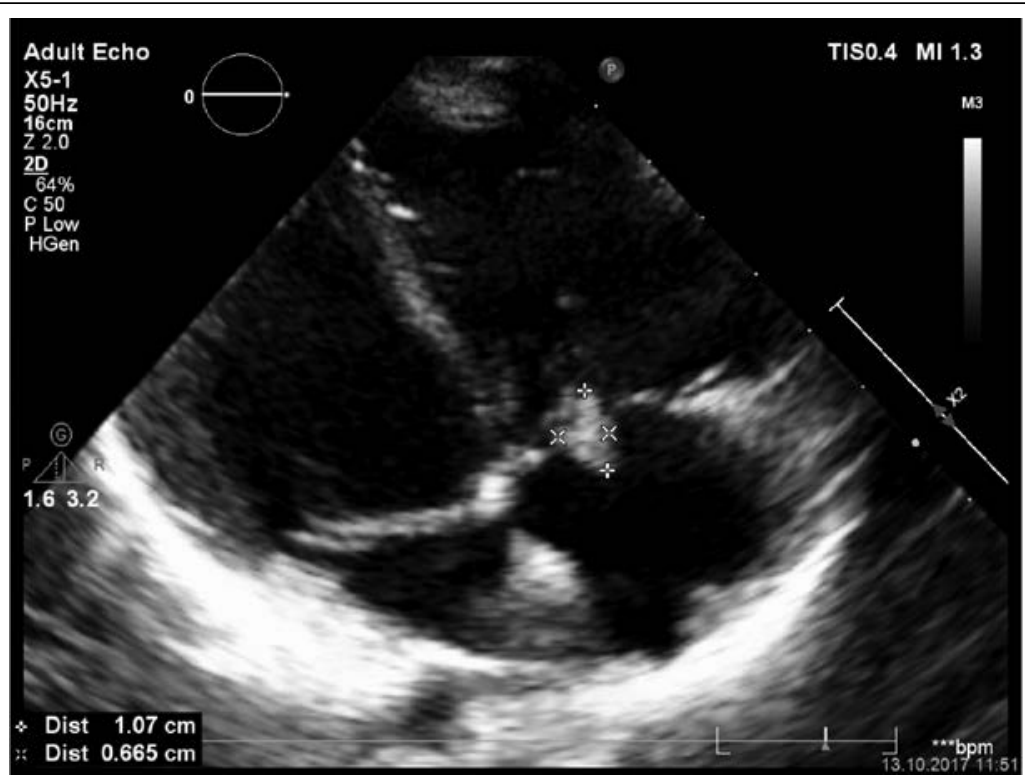

VIDEO CLIP 1. Ventricular septal defect (3 $\mathrm{mm}$ in diameter)

Link: http: / / www.sap.org.ar/uploads/archivos /general/files_855-moving-video_1mp4_1565371460.mp4 


\section{DISCUSSION}

Neurologic complications of IE significantly were associated with $S$. aureus vegetations and, frequently result of embolization from mitral or aortic valve vegetation. Ischemic, haemorrhagic events, sepsis, meningitis, and cerebral vasculitis are common clinical conditions. Multiple septic emboli may cause brain abscess or meningoencephalitis characterized by fever, headache, and meningismus. Aseptic or bacterial meningitis occur as a complication of IE at an estimated frequency of $3.5 \%$. Meningitis is usually purulent when associated with virulent organisms such as $S$. aureus. The CSF may present an aseptic formula when associated with subarachnoid hemorrhage or multiple microscopic embolic lesions. ${ }^{7,8}$ Despite the use of antibiotic agents, mortality remains high. Neurologic complications may be requiring cardiac surgery. ${ }^{9}$

Right-sided endocarditis accounts for $10 \%$ of all cases of IE. The most common presentation is persistent fever, bacteremia, multiple pulmonary emboli, pulmonary infarction, and right heart failure. Lamblin et al, reported 8-year-old boy with tricuspid valve infective endocarditis presented with multiple pulmonary abscess, pneumothorax and osteoarthritis. ${ }^{10}$ In the presence of VSD, vegetations can be found on the right ventricular side of the defect or on the tricuspid valve. Unlike left-sided endocarditis, neurologic and peripheral embolic manifestations are usually absent. If these manifestations are present, either left sided endocarditis or paradoxical embolism should be considered. Although few cases of right-sided IE have been reported with neurological symptoms in adults, children have not been described in literature. Lucas et al reported 24 adult patients who were diagnosed as infective endocarditis and bacterial meningitis. Only one patient had tricuspid valve endocarditis and meningitis due to $S$. aureus. ${ }^{11-13}$

Exclusive antibiotic treatment in IE may be insufficient. Surgery should be considered in the event of cardiac failure or persistent bacteremia refractory to treatment, severe valve failure, in large vegetations or in the event of major embolic phenomena. ${ }^{14}$

To our knowledge, right-sided IE appearing with meningeal signs have not been reported in children. Our patient admitted with clinic of aseptic meningitis, but she has not experience any neurological deficit and, we could not find any septic embolization clues on cranial imaging.
Recurrences of fever and known congenital heart disease, as well as echocardiographic examination lead to the diagnosis of infective endocarditis. In our patient aseptic meningitis is thought to result from small embolies, parameningeal inflammation, or low CSF bacterial burden associated with paradoxical embolization. Nonspecific neurological signs, like headache, with fever may be first clue of IE. Echocardiography should be performed in time to exclude IE in children with unexplained fever especially with congenital cardiac disease. Early diagnosis and treatment is important for minimize cardiac and neurological morbidities.

\section{REFERENCES}

1. Rosenthal LB, Feja KN, Levasseur SM, Alba LR, et al. The changing epidemiology of pediatric endocarditis at a children's hospital over seven decades. Pediatr Cardiol. 2010;31(6):813-20.

2. Marom D, Ashkenazi S, Samra Z, Birk E. Infective endocarditis in previously healthy children with structurally normal hearts. Pediatr Cardiol. 2013;34(6):141521.

3. Sun LC, Lai CC, Wang CY, Wang YH, et al. Risk factors for infective endocarditis in children with congenital heart diseases - A nationwide population-based case control study. Int J Cardiol. 2017;248:126-30.

4. Singh Y, Ganjoo N. Infective endocarditis in children. Paediatr Child Health (Oxford). 2017;27(2):68-74.

5. Mocchegiani R, Nataloni M. Complications of infective endocarditis. Cardiovasc Hematol Disord Drug Targets. 2009;9(4):240-8.

6. Venkatesan C, Wainwright MS. Pediatric endocarditis and stroke: a single-center retrospective review of seven cases. Pediatr Neurol. 2008;38(4):243-7.

7. Venkatesan A, Spalding C, Speedie A, Sinha G, Rumbaugh JA. Pseudomonas aeruginosa infective endocarditis presenting as bacterial meningitis. J Infect. 2005;51(4):e199202.

8. Aminoff MJ, Josephson SA. Neurological complications of systemic disease: adults. In Daroff R, Jankovic J, Mazziotta J, Pomeroy S. Bradley's Neurology in Clinical Practice. 7th ed. Philadelphia, PA: Elsevier Saunders; 2015.814-34.e1.

9. Heiro M, Nikoskelainen J, Engblom E, Kotilainen E, et al. Neurologic manifestations of infective endocarditis: a 17-year experience in a teaching hospital in Finland. Arch Intern Med. 2000;160(18):2781-7.

10. Lamblin A, Derkenne C. A child with fever, cough and Lancisi's sign. Pan Afr Med J. 2018;8;30:14.

11. Moss R, Munt B. Injection drug use and right sided endocarditis. Heart. 2003;89(5):577-81.

12. Lucas MJ, Brouwer MC, van der Ende A, van de Beek D. Endocarditis in adults with bacterial meningitis. Circulation. 2013;127(20):2056-62.

13. Williams LS, Allen BL. Neurolojic manifestations in Infective Endocarditis. In: Aminoff MJ, Josephson SA (eds). Aminoff's Neurology and General Medicine. 5thed.London: Elsevier; 2014:99-117.

14. Escarrá F, Fedullo AG, Veliz N, Rosa J, et al. Endocarditis por Streptococcus pneumoniae en pediatría: presentación de un caso clínico. Rev Chil Pediatr. 2017;88(6):776-80. 